\title{
Informatics-guided procurement of patient samples for biomarker discovery projects in cancer research
}

\author{
K. Stephen Suh · Yvonne K. Remache - Jalpa S. Patel • \\ Steve H. Chen · Russell Haystrand · Peggy Ford • \\ Anadil M. Shaikh · Jian Wang · Andre H. Goy
}

Received: 18 September 2008/Accepted: 15 October 2008/Published online: 19 November 2008

(C) The Author(s) 2008. This article is published with open access at Springerlink.com

\begin{abstract}
Modern cancer research for biomarker discovery program requires solving several tasks that are directly involved with patient sample procurement. One requirement is to construct a highly efficient workflow on the clinical side for the procurement to generate a consistent supply of high quality samples for research. This undertaking needs a network of interdepartmental collaborations and participations at various levels, including physical human interactions, information technology implementations and a bioinformatics tool that is highly effective and user-friendly to busy clinicians and researchers associated with the sample procurement. Collegial participation that is sequential but continual from one department to another demands dedicated bioinformatics software coordinating between the institutional clinic and the tissue repository facility. Participants in the process include admissions, consenting process, phlebotomy,
\end{abstract}

K. Stephen Suh $(\varangle) \cdot$ Y. K. Remache ·

J. S. Patel · R. Haystrand · P. Ford - A. M. Shaikh ·

A. H. Goy $(\bowtie)$

Tumor Bank and Genomics Program, The John Theurer

Cancer Center, Hackensack University Medical Center

Center, Hackensack, NJ 07601, USA

e-mail: ksuh@humed.com

A. H. Goy

e-mail: agoy@humed.com

S. H. Chen · J. Wang

BioFortis, Inc., 9017 Red Branch Rd Suite 103, Columbia, MD 21045, USA surgery center and pathology. During this multiple step procedures, clinical data are collected for detailed analytical endpoints to supplement logistics of defining and validating the discovery of biomarkers.

Keywords Tissue bank - Tumor .

Cancer · Procurement - Transbioinformatics ·

Bioinformatics · Workflow · Tissue procurement . Biomarker discovery

\section{Introduction}

Working with high-quality human tumor and tissue samples is essential for advancement of biomarker discovery program in modern cancer research (Bast et al. 2005). For this purpose, tissue repository or tumor banks are in need for research institutions, specializing in collecting human biomaterials and making them available for a wide array of research community at the level of institution, national or global. Several issues regarding the tissue repository facilities are currently undergoing refinements to maintain high quality clinical samples ${ }^{1,2}$ (Ericsson et al. 2006). Some of the major problematic issues

\footnotetext{
${ }_{1}$ National Cancer Institute Best Practices for Biospecimen Resources http://biospecimens.cancer.gov/global/pdfs/NCI_ Best_Practices_060507.pdf.

2 The Human Tissue Authority http://www.hta.gov.uk/.
} 
Table 1 Departments and their functions involved in the tissue repository flow

\begin{tabular}{|c|c|c|}
\hline & Departments & Functions \\
\hline A & Outpatient clinic & $\begin{array}{l}\text { Patients are consented by } \\
\text { the research nurse coordinator }\end{array}$ \\
\hline B & Outpatient laboratory & Blood drawn by phlebotomist \\
\hline $\mathrm{C}$ & Courier & $\begin{array}{l}\text { Transport samples from outpatient } \\
\text { laboratory to tissue repository }\end{array}$ \\
\hline $\mathrm{D}$ & $\begin{array}{l}\text { Surgical procedure } \\
\text { room }\end{array}$ & Disease tissue resected \\
\hline $\mathrm{E}$ & $\begin{array}{l}\text { Department of } \\
\text { pathology }\end{array}$ & $\begin{array}{l}\text { Gross samples fro diagnosis and } \\
\text { remaining bio-material procured }\end{array}$ \\
\hline $\mathrm{F}$ & $\begin{array}{l}\text { Information } \\
\text { technology }\end{array}$ & Automation and network support \\
\hline G & Tissue repository & Biomarker discovery \\
\hline
\end{tabular}

involved in the sample acquisition or procurement processes that are recognized by scientific communities and institutions are sample preparation, regulatory compliance (van Veen 2006), storage and processing. To use these samples in research, institutional studies and collaborations need to be evaluated by the institutional review board (IRB) and conform to Health Insurance Portability and Accountability Act (HIPAA).

Among the issues mentioned, one of the most difficult and critical parts to oversee and manage is the clinical workflow for sample acquisition, which starts from the time a patient is diagnosed and consented at the clinic to a point where the patient samples are finally passed on to the researchers at the tissue repository site (Table 1). Contributing factors affecting the workflow and eventual sample loss or questionable quality of sample include ineffective communication, lack of a centralized data management $^{3}$ (Patel et al. 2006) and a complex patientsample navigation system as a result of the multidisciplinary nature of the procurement process (Fig. 1). Effective control and management of the clinical workflow permits the coordinator of the biomaterial repository facility to oversee all steps involved in the tissue procurement and rapidly implement appropriate corrective actions when necessary. An efficiently operated workflow is critical to obtain high quality clinical samples and maintain consistent supply of patient samples in large numbers.

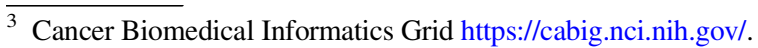

To incorporate solutions within each clinical steps of the workflow, our laboratory has used bioinformatics software Labmatrix ${ }^{\mathrm{TM}}$ (BioFortis, Inc., Columbia, MD) to guide the procurement of clinical samples. This product was chosen due to its (1) flexibility encompassing a diverse range of humanbased research areas, (2) support for workflows in typical clinical and research environments, (3) ability to seamlessly integrate multiple clinical and laboratory data streams, (4) robust programmatic interfaces enabling easy integration with other existing systems (e.g. Java toolkits and HL-7 transmission protocols), and (5) compliance to HIPAA standards and institutional security requirements.

Labmatrix was originally implemented at the tissue bank to digitally manage information on patient data and samples in order to replace paper laboratory notebooks and other ad hoc system (e.g. Excel and Access). Our laboratory has since customized the software based on our needs to build a remoteaccessible platform that facilitates multiple steps involved in the workflow within our medical center. This paper will focus on the implementation of the realtime and web-based workflow program that is enhanced by automated messaging and alert systems whenever a certain data condition is met. Combination of standardized clinical operational procedures and software automation enables us to do (1) electronic patient consenting, (2) patient specific clinical data entry, (3) efficient patient sample retrieval from phlebotomy department, surgical procedure rooms (e.g. Operating Room, OR) and pathology department, (4) accurate sample labeling, storage tracking and biobanking facilitation, (5) perpetual knowledge retention of sample history and experimental results, and (6) complete audit trail for all user activities.

\section{Bioinformatics guided workflow: solution to a tissue bank's problems and challenges}

Upon analysis of the original procurement workflow, one of the crucial problematic factors encountered in each step was ineffective communication. Poor communication lines all lead to confusion, eventual sample loss, and compromising sample integrity and quality. A lack of a centralized data management made it difficult to access and tracks informed consents and scheduled procedures. Moreover, we 


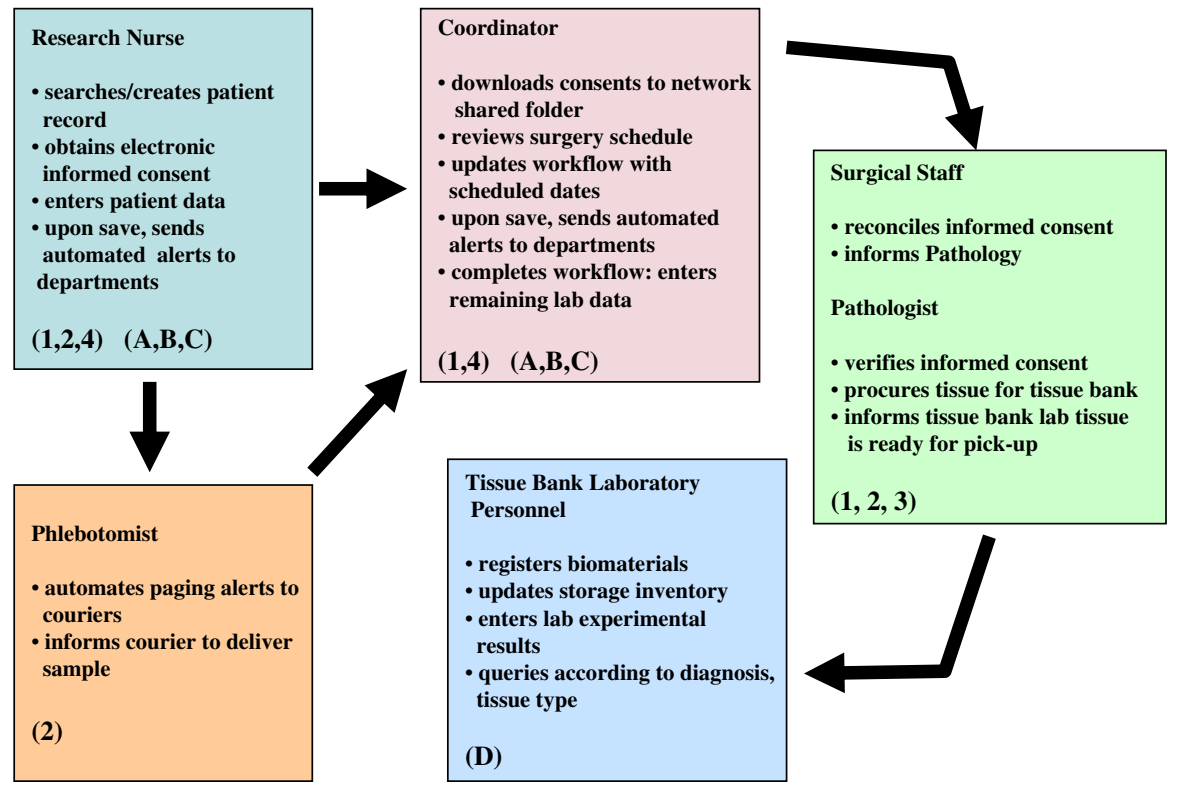

Fig. 1 Workflow for procurement of clinical biomaterials supported by bioinformatics, IT automation and designated coordinator of tissue repository. Different colors are assigned to each blocks to represent various departments involved in the procurement process, including Research nurse (RN),

were challenged with an inevitable complex patientsample navigation system which required interdisciplinary participation. Other factors consisted of a spatial issue causing electronic inaccessibility and a short-staffed clinic environment with a high patient volume. To resolve these workflow problems, Labmatrix $^{\mathrm{TM}}$ was customized to virtually oversee the sample procurement process.

\section{Problem 1: ineffective communication leading to inadequate sample acquisition}

Previously, tissue bank relied only on an electronic mailing system, comprised of patient identifiers, diagnosis, profile status, type of tissue and procedure patient had consented for. Lack of real time communication channels and access to consent documents contributed to significant sample loss as well as questionable quality of the samples.

To resolve this communication bottleneck, we implemented a bioinformatics guided workflow with many steps automated and taking advantage of information technology tools (Table 2). This workflow coordinator, phlebotomist, surgical staff/pathologist and tissue bank laboratory personnel. Numbers and Letters in the parenthesis in each box represent problems and solutions (Table 2) and snapshots of Labmatrix (Fig. 2) that are associated with the workflow

aims to bridge any communication gap by informing the tissue repository site, the surgery center and Pathology in advance without interrupting individual department's workflow. Once the patient is consented, an electronic signature will be obtained and saved into Labmatrix by the research nurse (Fig. 2a). The research nurse will now enter significant patient information in "Initial Consultation Form" in Labmatrix (Fig. 2b). Upon saving, an automated e-mail will be sent from Labmatrix to tissue repository site and Pathology, alerting that a patient has consented and to expect a sample if it is collected on that same day (Fig. 2c).

An automated e-mailing system along with a specific query building feature was integrated into Labmatrix for tracking and following-up future pending surgery dates and blood draw dates for consented patients. The coordinator will review the list of patients' names and compare with the surgical procedure schedule. Afterwards, the coordinator will take the necessary steps to update and save the pending surgery dates into Labmatrix. Upon save, an automated e-mail will be sent to the respective departments. 
Table 2 The table depicts problematic issues that tissue repositories encounter during the workflow and solutions to specific issue by installing a combination of IT automation and bioinformatics

\begin{tabular}{llc}
\hline & Problems & Solutions \\
\hline 1 & $\begin{array}{c}\text { Sample loss due to lack of communication } \\
\text { \& information }\end{array}$ & $\begin{array}{c}\text { All Communication and information are facilitated } \\
\text { by Labmatrix and (IT) automation. } \\
\text { Key events are electronically tracked through } \\
\text { a centralized data management. }\end{array}$ \\
3 & $\begin{array}{c}\text { Structural integrity and quality of biomaterials } \\
\text { compromised due to lack of coordination } \\
\text { Disruption of diagnostic routines in Pathology due to } \\
\text { unexpected sample arrival without consent forms }\end{array}$ & $\begin{array}{c}\text { Digitized informed consents and relevant legal documents } \\
\text { are accessible by authorized personnel via hospital } \\
\text { network. }\end{array}$ \\
& $\begin{array}{c}\text { IT communication not frequently accessible due to high } \\
\text { patient volume and short staff }\end{array}$ & $\begin{array}{c}\text { Virtual workflow, IT automation, and the designated } \\
\text { coordinator maximizes procurement efficiency. }\end{array}$ \\
\hline
\end{tabular}
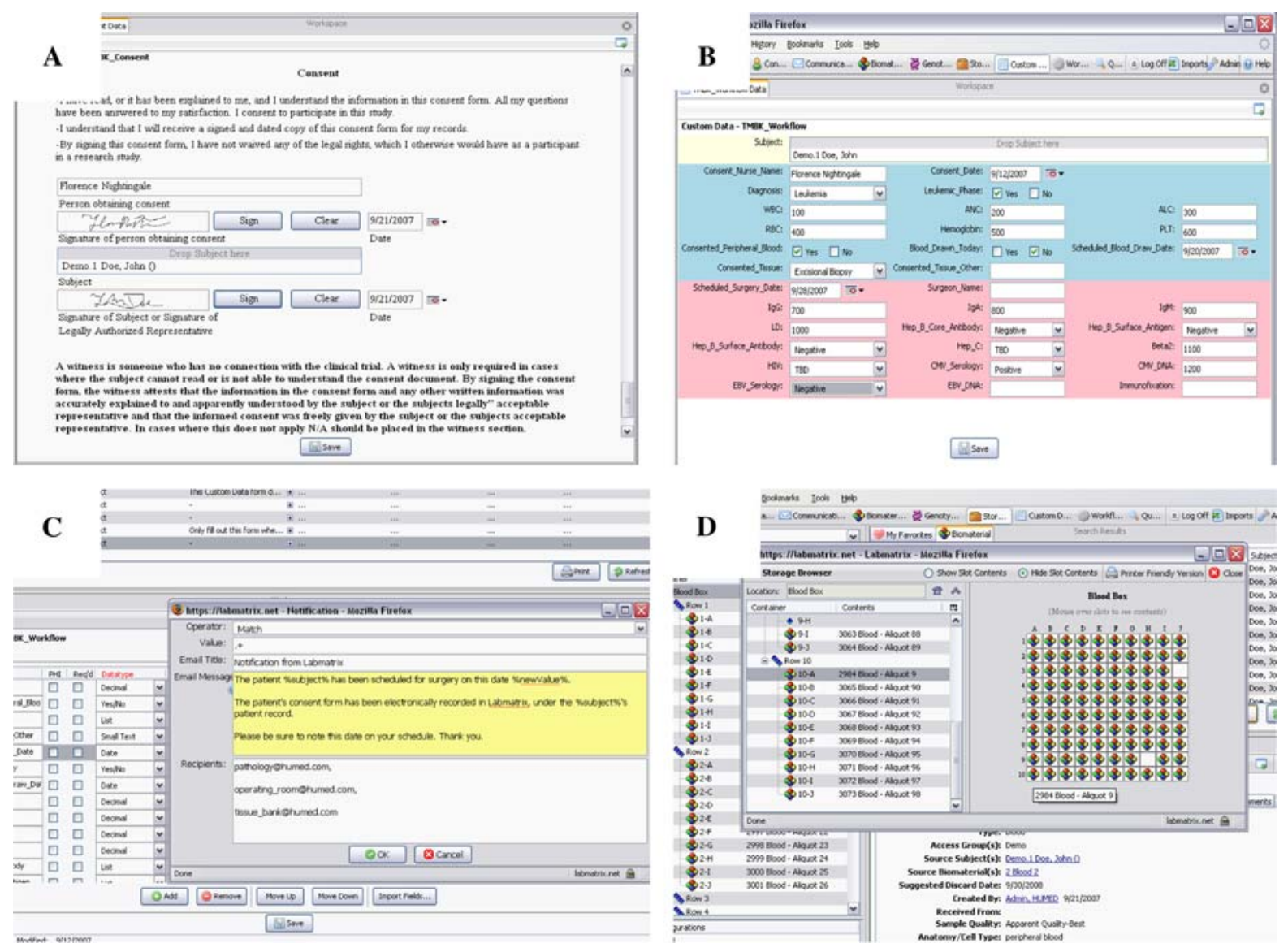

Fig. 2 Snapshots of Labmatrix interface representing a An electronic patient consent form, b A initial patient consultation and data forms, $\mathbf{c}$ IT automated e-mail alerts to relevant departments and d Storage tracking of biomaterials in the laboratory

\section{Problem 2: lack of centralized data management leading to difficulty in tracking consents and patient samples}

An overwhelming schedule in a fast-paced and stressful environment in the clinic resulted in an inefficient centralized data management, including inaccessibility to patient information and consents. The significance of the informed consent is that the patient is voluntarily giving permission to procure clinical samples for research. For instance, a signed consent is the document that Pathology must receive in advance. 
To facilitate and expedite tracking consent documents, the web-based workflow provides an electronic version of the signed consents, which are saved and documented in Labmatrix. At the end of the day, the coordinator will download and transfer the consent to a shared file in a predefined location in the network shared drive so that tissue bank, surgical staff members and Pathology can easily access the consent electronically. The consent will be renamed according to IRB and HIPAA approved format: corresponding medical record number (MRN)_patient initials_type of document. Renaming the file with patient's MRN will make the search simple and time efficient.

\section{Problem 3: complex patient/sample navigation}

The procurement process has two main sources: clinic and pathology. The procurement process is interdisciplinary, which may help explain the complexity of this patient-sample navigation.

Nurse and phlebotomist

After consenting, the patient is directed to blood collection lab and presents the require "Add-On Form". Occasionally, the form does not indicate to collect blood for tissue bank, resulting in sample loss. To ensure an effective communication between the research nurse and the phlebotomist, a copy of the consent is printed along with an "Alternative Reminder Form" which is a reminder for the nurse to indicate "Tissue Bank" on the "Add-On Form".

\section{Phlebotomist to courier}

Ineffective communication between the nurse, phlebotomist and couriers resulted in delayed sample delivery, thus compromising sample integrity and quality. Two common scenarios were encountered: (a) the courier picking up the note, but finding no sample in the refrigerator; and (b) the courier finds no note in the basket, but a sample was collected. In order to receive blood in a timely manner, the phlebotomist will store the collected samples in an insulated ice-packed bag to preserve the integrity of the sample. Furthermore, the phlebotomist will dial to activate automated-page. This automated-page will alert the charge nurse and the tissue repository site indicating that the blood is ready for pick-up. The charge nurse will assign the next available courier to deliver sample from phlebotomy to tissue repository site. If the blood is not received within an hour, the coordinator will investigate.

Surgery room to pathology to tissue bank

One common problem that arose between the surgery room to pathology and pathology to tissue bank concerned the availability of the patient's informed consent. Oftentimes, the tissue bank laboratory and Pathology were not informed that a patient had consented to tissue procurement, yet the surgical team was aware. Such scenario interrupted daily workflow for each department since members had to 'interrupt' their daily routine procedures to track down consent documents. This communication gap led to major sample loss. To overcome this communication issue, the coordinator will access and review the surgical procedure schedule on a daily basis and update surgery scheduled dates on the initial consultation workflow in Labmatrix. Once updated, an automated e-mail will be sent to the tissue repository site, surgical procedure staff and pathology.

The final step is sample retrieval from pathology to tissue repository site. Once fresh tissue and frozen tissue blocks or cassettes are ready for pick-up, Pathology will contact tissue repository facility via phone call. Tissue repository personnel will pick up fresh tissue on ice and frozen cassettes on dry ice-to protect and preserve sample integrity. The date and time will be recorded upon sample arrival for statistical purpose. The cassettes will be stored at $-80^{\circ} \mathrm{C}$ and the fresh tissue will be processed promptly and accordingly. Tissue bank personnel will register patient and biomaterial, generate barcodes, and update storage information onto Labmatrix (Fig. 2d). Once clinical lab results become available, the coordinator will enter the lab values into Labmatrix completing the tissue repository workflow.

\section{Discussion}

Translational bioinformatics is still a new field for both academic and commercial sectors. To date, most activities in this area focus on either data management 
or analysis. On the other hand, workflow support has been shown very effective, in many industries, to increase the quality and efficiency of repeatable processes. Clinical sample procurement is largely a repeatable process. We have demonstrated the value of translational informatics guided workflow supporting a tissue bank in a clinical environment. We believe workflow support will become increasingly important as a sub-specialty in translational bioinformatics as the field of translational research matures and becomes more standardized.

Open Access This article is distributed under the terms of the Creative Commons Attribution Noncommercial License which permits any noncommercial use, distribution, and reproduction in any medium, provided the original author(s) and source are credited.

\section{References}

Bast RC Jr, Lilja H, Urban N, Rimm DL, Fritsche H, Gray J, Veltri R, Klee G, Allen A, Kim N, Gutman S, Rubin MA, Hruszkewycz A (2005) Translational crossroads for biomarkers. Clin Cancer Res 11(17):6103-6108. doi: 10.1158/1078-0432.CCR-04-2213

Ericsson C, Franzen B, Nister M (2006) Frozen tissue biobanks. Tissue handling, cryopreservation, extraction, and use for proteomic analysis. Acta Oncol 45(6):643-661. doi:10.1080/02841860600818047

van Veen Ben-Evert (2006) Human tissue bank regulations. Nat Biotechnol 24:496-497. doi:10.1038/nbt0506-496

Patel AA, Gilbertson JR, Parwani AV, Dhir R, Datta MW, Gupta R, Berman JJ, Melamed J, Kajdacsy-Balla A, Orenstein J, Becich MJ (2006) An informatics model for tissue banks-lessons learned from the cooperative prostate cancer tissue resource. BMC Cancer 6:120. doi:10.1186/1471-2407-6-120 